\title{
Similarity Criteria, Galactic Scales, and Spectra
}

\author{
Georgy S. Golitsyn \\ A. M. Obukhov Institute of Atmospheric Physics, Russian Academy of Sciences, Moscow, Russia \\ Email: gsg@ifaran.ru
}

Received August 7, 2012; revised September 6, 2012; accepted September 13, 2012

\begin{abstract}
An old topic of dimensional analysis in astrophysics is presented and new results, or quantitative explanations of some observational facts are obtained, in particular, on the base of the supernova, SN, explosions. The presentation starts with the derivation of two similarity criteria for astrophysical objects constructed out of four measurable quantities: mass, $M$, luminosity, $L_{b}$, velocity $U$, size $R$, and gravitational constant $G$. The first well known criterium describes the virial principle and the other one seems to be new and is based on the Tully-Fisher observational relationship between luminosity and velocity. The energy generated by SN explosions allows one to estimate well the interstellar turbulent velocities and magnetic field in our Galaxy, resulting in 3 to 4 microgauss. It is found that for $z \geq 0.6$ the observed distant galactic clusters are far from virial equilibrium and the degree of disequilibrium is increasing with $z$. It means that to reach such an equilibrium the cluster age should be of order ten dynamical time scales, see Equation (7). For all considered galaxy clusters the second similarity criterium was found to be constant with a precision of about ten per cent. Therefore it could be considered as a general law, though for different classes of objects the numerical coefficient may vary. Some scales are proposed and two of them are tested for galactic clusters by finding numerical coefficients with accuracies of about 20 percent or better: e.g. observed luminocities of clusters are $W=L_{b}=a_{1}(M / R)^{5 / 2} G^{3 / 2}$ with $a_{1}=1.25 \pm 0.22$ for the first eleven objects from the Table for which the virial equilibrium is found with the same accuracy. The square root of the two criteria ratio $\Pi_{3}=\left(\Pi_{2} / \Pi_{1}\right)^{1 / 2}=U(W G)^{-1 / 5}$ explains the Tully-Fisher law and is constant for all 32 available clusters from [1,2] and is equal to $1.8 \pm 0.2$. This is because $\Pi_{3}$ has not global values of total mass and size.
\end{abstract}

Keywords: Dimensional Analysis; Galaxy Clusters; Virial Evolution; Tully-Fisher Law; Turbulence and Magnetic Fields

\section{Introduction}

Dimensional relationships between the measured quantities [3-7] are the base of studies of complex phenomena, certainly for astrophysics. Here we present such an analysis for quantities measured in astrophysics, find similarity criteria they may form, elucidate their meaning and possible applications, and present certain scales some of which are new ones, or at least used rarely. This analysis systemizes our knowledge of this methodology, presents some new results and may serve at least a methodological and educational purpose.

For our Galaxy we estimate turbulent gas velocities and magnetic fields in interstellar medium basing on the power supplied by supernova, SN, explosions. At distances $z>0.6$ galactic clusters are found to be far from the virial equilibrium between kinetic and potential energies and the degree of disequilibrium increases with $z$. This is in contrast with what this author was told by some professionals less than ten years ago that the virial equi- librium must not to be questioned. This believe prevented this author from exploring the subject for many years, through recently another authority in cosmology and astrophysics told me that the absence of such an equilibrium for early objects is selfevident. How this processes evolves is shown in this paper. Thanks to the invitation to present a paper for this Special Issue of the magazine this text has been written with a methodological goal to renew interest for the old subject which has been so productive in older times.

In astrophysics the measured quantities are mass, $M$, velocity, $V$, luminosity, $M$ or $L_{b}$, and sizes, $R$ (in absolute units, if distances to objects are known). Gravity fields are characterized by the constant $G=6.672 \times 10^{-12}$ $\mathrm{m}^{3} \cdot \mathrm{kg}^{-1} \cdot \mathrm{s}^{-2}$. Distances in astrophysics are measured in parsecs: $1 \mathrm{pc}=3.08 \times 10^{16} \mathrm{~m}=3.26$ light years. Our four measured quantities plus the gravity constant $G$ are determined by three independent dimensions: mass, length, and time. It follows from here that from these five quan- 
tities one can form two dimensionless values, the similarity criteria [3-7]. We choose the following as such criteria:

$$
\begin{aligned}
& \Pi_{1}=\frac{M G}{R U^{2}}, \\
& \Pi_{2}=\frac{M G^{3 / 5}}{R W^{2 / 5}} .
\end{aligned}
$$

The first value may be naturally called the virial criterium because it is formed by quantites entering the virial theorem applied to the central gravity field. It is belived that after sufficient time this theorem is true for an arbitrary number of interacting bodies. Then $\Pi_{1}=1$, if we would know with good precision all quantities entering (1), aside $G$, which we know with a relatively great precision. If $\Pi_{1} \neq 1$ it would demonstrate inaccuracies in our knowledge of masses, velocities, orbital and/or turbulent ones, negligence of some factors, e.g. dark matter or energy, an incompleteness of virialization of sufficiently large size objects, etc. Our Table below will demonstrate this for clusters of distant galaxies.

The second similarity criterium for the clusters is of order unity. This demonstrates a close connection among the quantities entering it, i.e. mass, size and brightness united by the gravity field. To this author's knowledge such a criterium have not yet been introduced. The long-known Tully-Fisher [8] relationship is a hint of such a connection:

$$
W \propto U^{n}, 3<n \leq 5,
$$

The upper limit for the exponent $n$ is an immediate consequence from these variables dimensions:

$$
W=U^{5} G^{-1}
$$

where from the velocity scale is

$$
U=(W G)^{1 / 5} .
$$

Numerical constants entering (4) and (5) should be determined experimentally and are expected to be of order unity as was noted by P. Bridgman [4] referring to Albert Einstein. Exponents $n$ lesser than 5 are evidenc that other similarity criteria act in the determination of the velocity scale (see [7]). e.g. if we take

$U=\left(\Pi_{1}^{-1} W G\right)^{1 / 5}$ we obtain $W \propto U^{3}$. From Equations

(1), (2) and (4) one may form the nondimensional similarity criterium as

$$
\Pi_{3}=\left(\Pi_{2} / \Pi_{1}\right)^{1 / 2}=c_{1} U(W G)^{-1 / 5},
$$

where $c_{1}$ is a numerical coefficient (see the end of Section 5), or $\Pi_{3}=c_{1}^{-1}$. The case that three quantities with three independent dimensions are forming a nondimensional criterium is a special case revealing a kind of the phenomenon degeneration. The only other case well known is the fine structure constant $e^{2} / \hbar c=1 / 137$ causing some conceptual problems in quantum electrodynamics. Such situations are discussed by P. Bridgman [4] in Chapter 8.

Knowing the size and the velocity scale we determine the time scale from (1):

$$
T_{d}=\frac{R}{U}=\left(\frac{R^{3}}{M G}\right)^{1 / 2},
$$

which with the mass density scale $\rho=M R^{-3}$ equals to

$$
T_{d}=(G \rho)^{-1 / 2} \text {. }
$$

The last time scale, obvious from dimensions of $G$ and $\rho$, can be found in the book by M. Rees [9] where it is called the dynamical time scale. It is compared there with the cooling time $T_{c}$ of the earlier gas by recombination, transfer of energy between various atomic levels, etc. leading to the energy emission and gas cooling. If $T_{c}<T_{d}$, then primordial gas cloud is either compressed to its center, or fragmented into smaller objects, which may lead to formation of galaxy clusters.

\section{Galactic Scales}

The observational situation is such that, all four quantities $W, U, M, R$ are very rarely determined simultaneously for the same object. At the same time if we measure only two values out of four, together with the gravity constant $G$ we may still form some useful scales.

As an example, let as choose mass $M$, size $R$ and $G$. Then the velocity scale is, as from (1):

$$
U=\left(M G R^{-1}\right)^{1 / 2},
$$

the time scale is

$$
T=2 \pi R^{3 / 2}(M G)^{-1 / 2}
$$

and $2 \pi$ is taken for the time of the full revolution of the object around the gravity center. The brightness, the power scale is

$$
W=M^{5 / 2} G^{3 / 2} R^{-5 / 2},
$$

the energy density in the unit volume, dimension of pressure, is

$$
w=M^{2} G R^{-4},
$$

and the power density per unit mass is

$$
\varepsilon=M^{3 / 2} G^{3 / 2} R^{-5 / 2} .
$$

For our Galaxy with $4 \times 10^{11}$ stars with the solar mass $M_{\odot}=2 \times 10^{30} \mathrm{~kg}$ and brightness $W_{\odot}=4 \times 10^{26} \mathrm{~W}$ we obtain $U=380 \mathrm{~m} / \mathrm{s}, T=240 \mathrm{My}, W=1.6 \times 10^{38} \mathrm{~W}$, $\varepsilon=1.6 \times 10^{-4} \mathrm{~m}^{2} \cdot \mathrm{s}^{-3}, w \approx 2 \times 10^{-9} \mathrm{~J} / \mathrm{m}^{3}$. First three scales 
are rather close to the observed ones. But the last value requires a comment.

It is known [10] that for our Galaxy the volume density of cosmic rays energy is close to

$0.5 \mathrm{eVcm}^{-3}=0.6 \times 10^{-13} \mathrm{~J} / \mathrm{m}^{3}$ The energy density of the galactic interstellar magnetic field is $H^{2} / 8 \pi \approx 10^{-12}$ $\mathrm{erg} / \mathrm{cm}^{3}=1 \times 10^{-13} \mathrm{~J} / \mathrm{m}^{3}$ at $H \approx 5 \times 10^{-6}$ Gauss. The kinetic energy density of turbulent gas motions are of the same order of magnitude, as well as the energy density of the star EM emissions and even the density of the relict emission [10]. The last could be a chance coincidence. At the same time Equation (11) gives a four magnitudes higher value. This may be explained by rewriting Equation (11) using (8) as $w=M U^{2} R^{-1}$ showing that it is the energy volume density of the large scale orbital motions. But we are interested in small scales.

The cosmic rays, $\mathrm{CR}$, and galactic magnetic fields are generated by SN explosions. SN explode in our Galaxy two-three times in a century and $10^{52}-10^{53}$ Jouls are generated at each explosion [10]. In Watts it corresponds to $3 \times 10^{33} \mathrm{~W}$. This and the CR energy volume density and direct use of the notion of the spectrum are enough to explain the observed shape of the CR spectrum [11,12]. With the velocity of light $c$ and the Stefan-Bolzmann constant $\sigma$ one may define the temperature scale $T_{g}$ using the volume energy density $w$ as:

$$
T_{g}=\left(\frac{3 w c}{4 \pi \sigma}\right)^{1 / 4},
$$

where $(4 \pi / 3)$ is the area of a unit sphere. With $w \approx 10^{-13} \mathrm{Jm}^{-3}$ we obtain $T_{g}=3.35 \mathrm{~K}$. This is close to relict temperature $2.7 \mathrm{~K}$. This is a single scale with the hint of the quantum mechanics because

$$
\begin{aligned}
& \sigma=\frac{\pi^{2}}{60} \frac{k^{4}}{h^{3} c^{2}}=5.67 \times 10^{-8} \mathrm{~W} \cdot \mathrm{L}^{-2} \cdot \mathrm{K}^{-4}, \\
& k=1.38 \times 10^{-23} \mathrm{~J} \cdot \mathrm{K}^{-1} .
\end{aligned}
$$

is the Bolzmann constant, $h=1.06 \times 10^{-33} \mathrm{~J} \cdot \mathrm{s}$ is the Planck constant.

M. Rees [9] has introduced the notion of virtual temperature as a measure of kinetic energy in the object of consideration. It is defined from Equation (1) at $\Pi_{1}=1$. Then

$$
T_{v}=\frac{m M G}{k R}=\frac{m}{k} U^{2},
$$

where $m$ is the mean atomic weight in the gas cloud. In its primordial composition protons are about 90 percent and helium is of order 10 percent. The mass of proton is $m_{p}=1.66 \times 10^{-27} \mathrm{~kg}$. Therefore in Equation (14) $\mathrm{m}=$ $1.9 \times 10^{-27} \mathrm{~kg}$.

Let us consider what these scales are for a star like our Sun with mass $M=2 \times 10^{30} \mathrm{~kg}$ and luminosity $4 \times 10^{26}$ $\mathrm{W}$. Then the linear scale determined by its mass and lu- minosity is

$$
R=M W^{-2 / 5} G^{3 / 5},
$$

and is equal to $3.6 \times 10^{13} \mathrm{~m}=240$ a.u., astronomic units. It seems that this was the size of the original cloud from which the Sun and the Solar system were formed. The mass of the cloud was several times larger than the mass of the Sun, therefore the size of cloud was accordingly larger as Equation (15) is demonstrating. The virial similarity criterium Equation (1) for the Sun at

$R=7 \times 10^{5} \mathrm{~km}=7 \times 10^{8} \mathrm{~m}$ is $2 \times 10^{11} U^{-2}$. At $\Pi_{1}=1$ we have $U=4.5 \times 10^{5} \mathrm{~m} / \mathrm{s}=450 \mathrm{~km} / \mathrm{s}$. We see that the virial criterium to the single star is not applicable. On the other hand a reasonable estimate is produced by Equation (5): $U=(W G)^{1 / 5}=2.3 \mathrm{~km} / \mathrm{s}$, which is characteristic for the convective velocities in the photosphere. One can write formulas for the scales as Equations (8)-(12) for any other couple of measured quantities like size $R$ and luminosity $W$, or mass and luminosity, etc. We see from the above examples that the purely dimensional analysis is still able to produce useful results, at least, of educational value. One may note a very high dependence of luminosity in Equation (10) on mass and size as $(M / R)^{5 / 2}$. It may explain the high luminosities of compact quasars. If we know only the luminosity $W$ we may estimate the ratio $M / R$ for the virialized objects as $a_{1} W^{2 / 5} G^{-3 / 5}$ which $a_{1}=0.93 \pm 0.20$ estimated from eleven first lines of our Table.

The similarity criterium $\Pi_{3}$ is found to be unexpectedly close to a constant value for all objects of our Table. The coefficient $a$ varies from 0.50 to 0.65 with the mean value and dispersion $a=0.56 \pm 0.04$ even accounting for the cluster No. 12 for which $a=0.34$. It makes us think that this cluster is somewhat unusual having a small mass at a comparatively high luminosity [1].

The addition of ten objects from [2] for which the value of $\Pi_{3}$ can be calculated does not change noticeably the value of $a=0.56$. Therefore the similarity criterium $\Pi_{3}=U(W G)^{-1 / 5}$ is equal to $a^{-1}=1.8 \pm 0.2$ for the galactic clusters. For other objects it may vary. Certainly the similarity criterium $\Pi_{3}$ is of more universal meaning than $\Pi_{1}$ since it does not depend on the global parameters of a system like its mass and size determining the system virial equilibrium between the kinetic and potential energies.

The paper [2] has some detailed information on 13 nearly clusters with $z \leq 0.23$. However only for ten of them the criteria $\Pi_{1}$ and $\Pi_{3}$ can be evaluated using the larger values for mass, size and temperature, see eq. (18). One can not directly compare such data with the parameters from our Table. Using what we have we compute the virial criterium $\Pi_{1}$ to be $0.44 \pm 0.04$ instead of 1 . This is mainly caused by the factor $M / R$, evidently by overestimating the size of an object which is 
still can be measured but contains only a small fraction of the total mass. The criterium $\Pi_{3}$ is close to $1.8 \mathrm{sub}-$ stantiating the results from the Table.

For the Sun at $T_{e}=5750 \mathrm{~K}, W=2 \times 10^{26} \mathrm{~W}$ at $a=$ 0.56 the value $\Pi_{3}=7$ For our Galaxy at $W=8 \times 10^{37}$ $\mathrm{W}$ as from $4 \times 10^{11}$ stars like Sun and at the mean orbital velocities $U=300 \mathrm{~km} / \mathrm{s}$ we find $\Pi_{3}=1.35$. It looks like that for similar single objects the similarity criterium $\Pi_{3}$ could vary.

\section{Turbulence of a Galactic Interstellar Gas}

Energy generation for the turbulent gas motions comes from the $\mathrm{SN}$ explosions. The power of this process is estimated above as $3 \times 10^{33} \mathrm{~W}$ for our Galaxy [10]. During explosions the shock waves tear off the external shells of SN and spread their matter from random places in the Galaxy and at random times. Random shock waves in space accelerate CR particles according to Fermi mechanism. We assume the mass of the interstellar gas as $M$ $=10^{40} \mathrm{~kg}$. This is two order of magnitude less than the gravity mass of the Galaxy where over 90 percent is the dark matter, i.e. the mass of the gas is an order magnitude less than the mass of the stars. The SN energy power for the unit gas mass will be $\varepsilon=3 \times 10^{-7} \mathrm{~m}^{2} \cdot \mathrm{s}^{-3}$. This is the energy rate of generation of the matter perturbation and in the statistically stationary case it will be also the rate of the energy dissipation.

Armstrong et al. [15] have shown that the spatial energy spectrum for the electron density fluctuations in the interstellar gas is proportional to $k^{-5 / 3}$ where $k=2 \pi / r$ is the spatial wave number up to distances $r=500 \mathrm{pc}=$ $1.5 \times 10^{19} \mathrm{~m}$. The velocity fluctuations have the same structure up to $100 \mathrm{pc}$ [10]. For the velocity fluctuations A. N. Kolmogorov have proposed in 1941 the structure function

$$
D_{u}(r)=\left\langle[u(x+r)-u(x)]^{2}\right\rangle=b(\varepsilon r)^{2 / 3}
$$

where the numerical coefficient $b$ is close to 2 as it was later obtained from a great number of various measurements (see [14]). With the above estimated $\varepsilon$ and $r=3 \times 10^{18} \mathrm{~m}=100 \mathrm{pc}$ we obtain $\langle\delta u\rangle=\left[D_{u}(r)\right]^{1 / 2}=20$ $\mathrm{m} / \mathrm{s}$, a value close to the observed velocity fluctuations in our Galaxy.

\section{Magnetic Field in the Galaxy}

To describe this field we determine the structure function using the same arguments as A. M. Obukhov has used in 1949 (see [1]) for the description of the statistical structure of a passive scalar. But instead of the magnetic field induction $H_{i}$ we shall do it for the modulus of the Alven velocity $V=H(4 \pi \rho)^{-1}$. Then

$$
D_{A}(r)=\left\langle\left[V_{A}(x+r)-V_{A}(x)\right]^{2}\right\rangle=N \varepsilon^{-1 / 3} r^{2 / 3},
$$

where the rate of magnetic energy generation/dissipation is defined as

$$
N=\left\langle\frac{\mathrm{d}}{\mathrm{d} t} \frac{V_{A}^{2}}{2}\right\rangle=\left\langle\rho \frac{\mathrm{d}}{\mathrm{d} t}\left(\frac{H^{2}}{8 \pi \rho}\right)\right\rangle .
$$

Since the volume density of the magnetic energy is the same as the energy of velocity fluctuations, see above, and both are due to SN explosions, the time scales for both quantities should be of the same order. Then $N \varepsilon^{-1 / 3} \approx \varepsilon^{2 / 3}=\left(3 \times 10^{-1} \mathrm{~m}^{2} \cdot \mathrm{s}^{-3}\right)^{2 / 3}=5 \times 10^{-5} \mathrm{~m}^{4 / 3} \cdot \mathrm{s}^{-2}$. For $r=100 \mathrm{pc}=3 \times 10^{18} \mathrm{~m}$ we will have from Equation (16) $V_{A}^{2}=10^{8} \mathrm{~m}^{2} / \mathrm{c}^{2}$. The mean density of the interstellar gas at $V=10^{60} \mathrm{~m}^{3}$ is equal to $M / V=\rho=10^{-24} \mathrm{~g} \cdot \mathrm{cm}^{-3}$, or of an order less than one proton on $\mathrm{cm}^{-3}$. Coming from the square of the Alven velocity to the magnetic field we obtain, at this density of matter, $H \approx 3.5 \times 10^{-6}$ Gauss. The observed values of the magnetic field in our Galaxy vary from 3 to 5 microgauss. Its fluctuations in space should also be described by the Kolmogorov-Obukhov $k^{-5 / 3}$ spectrum. Such spectra have been obtained for the magnetohydrodynamic turbulence by Frick and Sokolov [2].

The last two subsections, just described, relate well the observed velocity fluctuations and magnetic fields to the observed rate of SN explosions in our Galaxy.

\section{Clusters of Galactics and Their Similarity Criteria}

This author was a reviewer of A. A. Vikhlinin's D.Sc. Dissertation "The observational Cosmology and the Intergalactic Medium Studies by X-ray Spectra of Galactic Clusters" [1]. It has a detailed analysis of X-ray and optical data for 21 distant clusters at $z$ from 0.4 to 1.26 . A later paper [2] presents 13 relaxed closer clusters with $z \leq$ 0.23 and necessary references. Table 1 has all the data from [1] needed for the subsequent analysis. The last three columns are calculated by us.

In the Table $1 \mathrm{z}$ is the red shift, the difference between the length of the registered emission and emitted one related to the length of the emitted wave, temperatures $T$ in $\mathrm{keV}$ from 2.2 to $14 \mathrm{keV}$, i.e. from $2.5 \times 10^{7}$ to $18 \times 10^{7} \mathrm{~K}$, bolometric luminosity $W$ from 2 to 260 times $10^{37} \mathrm{~W}$, the mass is from 0.2 to 8.77 times $10^{14} M_{\odot}=$ $2 \times 10^{44} \mathrm{~kg}$, the spectral radius in Mpc, $1 \mathrm{Mpc}=3 \times 10^{22}$ $\mathrm{m}$. It was assumed that the gas velocities observed by the broadening of spectral lines are the thermal ones and the temperature can be restored from them as

$$
T=\frac{\mu m_{p} U^{2}}{3 k A},
$$


Table 1. Parameters of the distant clusters and their virial similarity criterium $\Pi_{1}$ (Equation (1)).

\begin{tabular}{|c|c|c|c|c|c|c|c|c|}
\hline № & $z$ & $T,(\mathrm{keV})$ & $W,\left(10^{37} w\right)$ & $M, 10^{14} M_{\odot}$ & $R, \mathrm{Mpc}$ & $\Pi_{1}$ & $T_{a}(\mathrm{By})$ & $T_{d}(\mathrm{By})$ \\
\hline 1 & 0.394 & 4.8 & 9.2 & 1.24 & 0.5 & 0.96 & 8.12 & 0.48 \\
\hline 2 & 0.400 & 3.7 & 8.9 & 1.42 & 0.7 & 1.02 & 8.09 & 0.49 \\
\hline 3 & 0.424 & 3.6 & 10.6 & 1.07 & 0.5 & 1.05 & 7.92 & 0.61 \\
\hline 4 & 0.426 & 7.6 & 27.0 & 2.89 & 0.9 & 0.79 & 7.91 & 0.50 \\
\hline 5 & 0.451 & 14.1 & 260.4 & 8.77 & 0.9 & 1.23 & 7.75 & 0.43 \\
\hline 6 & 0.453 & 5.8 & 15.9 & 1.81 & 0.7 & 0.83 & 7.73 & 0.65 \\
\hline 7 & 0.460 & 5.3 & 16.3 & 1.57 & 0.5 & 1.10 & 7.68 & 0.55 \\
\hline 8 & 0.516 & 5.1 & 15.7 & 1.67 & 0.6 & 1.02 & 7.34 & 0.54 \\
\hline 9 & 0.537 & 8.1 & 91.7 & 3.68 & 1.0 & 0.85 & 7.21 & 0.78 \\
\hline 10 & 0.541 & 9.9 & 113.3 & 6.43 & 1.0 & 1.21 & 7.19 & 0.59 \\
\hline 11 & 0.562 & 4.8 & 12.5 & 1.19 & 0.5 & 0.92 & 7.07 & 0.49 \\
\hline 12 & 0.574 & 2.7 & 38.8 & 0.36 & 0.5 & 0.50 & 7.00 & 0.88 \\
\hline 13 & 0.583 & 5.2 & 10.8 & 0.95 & 0.5 & 0.68 & 6.95 & 0.54 \\
\hline 14 & 0.700 & 7.2 & 28.7 & 2.01 & 0.7 & 0.74 & 6.36 & 0.62 \\
\hline 15 & 0.782 & 6.3 & 32,4 & 1.41 & 0.7 & 0.59 & 5.99 & 0.74 \\
\hline 16 & 0.805 & 2.2 & 2.0 & 0.21 & 0.5 & 0.36 & 5.89 & 1.16 \\
\hline 17 & 0.805 & 4.3 & 13.2 & 1.04 & 0.8 & 0.56 & 5.89 & 1.05 \\
\hline 18 & 0.813 & 6.6 & 28.8 & 1.25 & 0.7 & 0.50 & 5.86 & 0.79 \\
\hline 19 & 0.823 & 7.8 & 70.9 & 2.58 & 1.0 & 0.62 & 5.81 & 0.93 \\
\hline 20 & 1.100 & 3.5 & 5.9 & 0.26 & 0.5 & 0.28 & 4.82 & 1.04 \\
\hline 21 & 1.261 & 4.7 & 6.0 & 0.20 & 0.5 & 0.16 & 4.36 & 1.19 \\
\hline
\end{tabular}

$\mu=0.6$ is the mean molecular weight of plasma with the space concentration of protons and helium ions, electrons are taken into account, $m_{p} \approx 1.9 \times 10^{-27} \mathrm{~kg} \approx 2 \times$ $10^{-27} \mathrm{~kg}$ the mean mass of the gas particle, $k=1.38 \times 10^{-23}$ $\mathrm{J} / \mathrm{K}, \quad A=1.161 \times 10^{7} \mathrm{~K} / \mathrm{keV}$.

It is useful to compare the data from the Table with the corresponding parameters for our Galaxy with $R=15$ $\mathrm{kpc}, U=300 \mathrm{~km} / \mathrm{c}, M=4 \times 10^{11} M_{\odot}=10^{42} \mathrm{~kg}$. Then Equation (1) gives $\Pi_{1}=1.2$, close to unity, which is evidencing that our estimates of the parameters entering Equation (1) are reasonable.

Let us return to our Table 1. In one before the last column we estimate the object age by dividing the Universe age of 13.7 By by $1+z$ and extracting from the ratio 1.7 By, the estimate of time by Rees [9] when clusters could form. The last column contains the dynamic time $T_{d}=(G \rho)^{-1 / 2}$, Equation (7). This time scale gives a representation of the nature of the virialization dergee, i.e. a degree of dynamic equilibrium between kinetic energy of motions and gravity energy. The ratio of the two scales $T_{a} / T_{d}$ gives the cluster age in the time units of $T_{d}$.

For the first eleven objects the difference of the virial criterium $\Pi_{1}$ from unity is rather small and random, as for our Galaxy. These differences can be related to the measurements inaccuracies and/or to rounding errors. For the last ten objects $\Pi_{1}$ is systematically smaller than unity with the minimal value of $\Pi_{1}=0.16$ for the most distant object with $Z=1.26$. This corresponds to the age $4.36 \mathrm{By}$ at the scale $T_{d}=1.2 \mathrm{By}$. Evidently several, up to ten, units of $T_{d}$ are needed for reaching the virialization. The eleven objects from [2] all have the virial similarity criteria close to unity (spread about $20 \%$ or less) and do not add substantially new information to our Table 1. Figure 1 presents the values of criteria $\Pi_{1}$ in dependence on the ratio of their life time $T_{a}$ to the time of dynamic relaxation $T_{d}$. It is evident about $10 \mathrm{dy}-$ namical time scales of $T_{d}$ are necessary that for the virialization of these objects. It is interesting to note that in a dense gas one or two molecular collision times are 


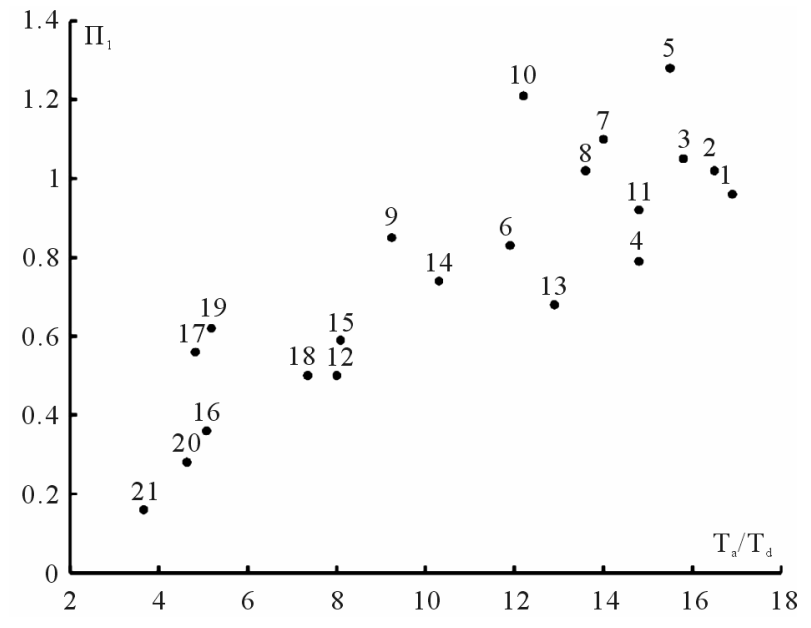

Figure 1. The virial similarity parameter $\Pi_{1}=M G / R U^{2}$ for the 21 galactic clusters from the table versus the ratio of the cluster age $T_{0}$ to its dynamic relaxation time $T_{d}=(\rho G)^{-1 / 2}, \rho$ being the cluster mass density.

needed for the Boltzmann thermal equilibrium, but in collisionless plasma [16] several dynamic time scales are needed as well for reaching an equilibrium among various degrees of freedom.

\section{Conclusions}

In astrophysics four quantities can be measured of an object: mass $M$, luminocity $W$, velocity $U$, size $R$. There is also the gravitational constant $G$, five quantities with dimensions out of three dimension units: mass, time, and length. Therefore two non-dimensional similarity criteria can be formed and various scales. We start to explain turbulent velocity and magnetic fields. For our Galaxy on the base of supernova explosions we explain the structure and intensity of these fields.

As the first similarity criterium we use the well known virial ratio between potential and kinetic energies: $M G / R U^{2}$. For 21 cluster of galaxies from [1] it is found that this ratio is close to unity for really clusters with redshift $z<0.6$. The 20 per cent scatter may be understood to measurement inaccuracies. The same is for 11 nearly clusters from [2]. The clusters [1] with $z>0.6$ have lesser and lesser degree of virial relaxation the younger they are. A notion of interaction time $T_{d}$ is introduced and it is concluded that of order $10 T_{d}$ time is needed to reach a relaxation for a cluster.

The second criterium $U(W G)^{-1 / 5}$ is found to be constant at $1.8 \pm 0.02$ for all 21 clusters from [1] including not yet relaxed ones and for 11 relaxed clusters from [2]. This unpexected finding needs a model explanation together with an old Tully-Fisher [8] relation for radiogalaxies that $W \sim U^{5}$.

The reasons for this paper to be written are exposed in the abstract and in the Introduction. I may add two re- frences for Wesson $[17,18]$ on the application of dimensional analysis to cosmology, which could be a good starting point in this direction. It should begin, as in any other case, with an analysis of what the problem is physically, what is measured and/or should be measured and how everything is interconnected. Of course, it would not solve the whole problem of cosmology but may reveal some important and/or interesting aspects.

\section{Acknowledgements}

As a student of Moscow University I visited in midfifties a special lecture course on astrophysics by Prof. A. I. Lebedinsky. My later work was in environmental physics in a broad sense, lately there were occasionally a few specific astrophysical problems, like cosmic rays energy spectrum, or analogy between earth- and star-quakes. Sporadic meetings and discussions with I. S. Shklovsky, Ya. B. Zeldovich, R. A. Syunyaev and some others kept alive my interest in astrophysics. I am grateful to all these people and their memories, though occasional talks with Rashid Syunyaev, Alexey Starobinsky and Alexey Vikhlinin still continue.

\section{REFERENCES}

[1] A. A. Vikhlinin, "Observational Cosmology and the Study of Intergalactic Medium by X-ray Data on Galactic Clusters," Institute for Cosmic Research, RAS, Moscow, 2002.

[2] A. A. Vikhlinin, et al., "Chandra Sample of nearby Relaxed Galaxy Clusters: Mass, Gas Fraction, and MassTemperature Relation," Astrophysical Journal, Vol. 640, No. 2, 2006, pp. 691-709. doi:10.1086/500288

[3] E. Buckingham, "On Physically Similar Systems," Physical Review, Vol. 4, No. 3, 1914, pp. 354-376.

[4] P. Bridgman, "Dimensional Analysis," Yale University Press, New Haven, 1931.

[5] G. Birkhoff, "Hydrodynamics, a Study in Logic, Fact, and Similitude," 2nd Edition, Princeton University Press, Princeton, 1960.

[6] L. I. Sedov, "Similarity and Dimensional Methods in Mechanics," Academic Press, New York, 1959.

[7] G. I. Barenblatt, "Scaling," Cambridge University Press, Cambridge, 2003. doi:10.1017/CBO9780511814921

[8] R. B. Tully and J. R. Fisher, "A New Method of Determining Distances in Galaxies," Astronomy and Astrophysics, Vol. 54, No. 3, 1977, pp. 661-673.

[9] M. Rees, "New Perspectives in Astrophysical Cosmology," 2nd Edition, Cambridge University Press, Cambridge, 2002.

[10] V. L. Ginsburg, "Astrophysics of Cosmic Rays," North Holland, Amsterdam, 1990.

[11] G. S. Golitsyn, "Cosmic Ray Spectrum from the Similarity Point of View," Astronomy Letters, Vol. 23, No. 2, 1997, pp. 321-325. 
[12] G. S. Golitsyn, "Phenomenological Explanation of the Spectrum of Cosmic Rays with Energies $E>10 \mathrm{GeV}$," Astronomy Letters, Vol. 31, No. 7, 2005, pp. 500-505.

[13] A. S. Monin and A. M. Yaglom. "Statistical Hydrodynamics," Vol. 2. MIT Publishing, Cambrisge, 1975.

[14] P. Frick and D. Sokolov, "Cascade and Dynamo Action in a Shell Model of Magnetohydrodynamic Turbulence," Physical Review E, Vol. 57, No. 4, 1998, pp. 4155-4164. doi:10.1103/PhysRevE.57.4155

[15] J. W. Armstrong, J. M. Cordes and B. J. Rickert, "Density
Power Spectrum in the Local Interstellar Medium," $\mathrm{Na}$ ture, Vol. 291, 1981, pp. 561-564. doi:10.1038/291561a0

[16] B. B. Kadomtsev, "Collective Phenomena in Plasma," Fizmatlit Publishing House, Moscow, 1976.

[17] P. Wesson, "Cosmology and Geophysics," D. Reidel. Dordrecht, Holland, 1978.

[18] P. Wesson, "The Application of Dimensional Analysis to Cosmology," Space Science Reviews, Vol. 27, No. 2, 1980, pp. 109-153. doi:10.1007/BF00212237 